\title{
XXIV. A new form of Influence-machine
}

\section{James Wimshurst}

To cite this article: James Wimshurst (1893) XXIV. A new form of Influence-machine, Philosophical Magazine Series 5, 36:220, 264-267, DOI: 10.1080/14786449308620474

To link to this article: http://dx.doi.org/10.1080/14786449308620474

$$
\text { 曲 Published online: } 08 \text { May } 2009 .
$$

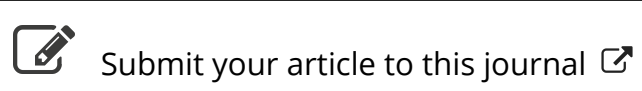

\footnotetext{
Џll Article views: 3
}

Q View related articles $\sqsubset$ 
But there is another ground on which this course is seen to be for him entirely illogical. For though his having taken it is an admission that he regards the proposition in question as incapable of deduction, he has been hasty in reaching this conclusion. We have seen that, in order to maintain complete transformation during tranference, he has confessedly to assume that bodies consist of massive particles without elasticity, and that the medium is elastic but without inertia. Now, as pointed out in my paper (p. 141) :- "That energy cannot be transformed without being transferred must of course be true if bodies consist of particles with inertia but without elasticity, and if the medium connecting them possess elasticity but not inertia." This is surely quite obvious. Hence with the assumptions already made by Prof. Lodge the proposition under consideration is capable of deduction. For bim therefore it cannot be an axiom.

For those of us who do not hold to his theory of the constitution of bodies and media, the proposition is of course not capable of deduction. Whether or not we are to regard it as axiomatic must depend on whether or not it may be shown to be capable of coordinating dynamical phenomena generally.

Edinburgh, July 21st, 1893.

\section{A New Form of Influence-Machine. By James Wimshurst*}

TN April 1891 I had the honour to submit to this Meeting a very useful form of experimental Influence-machine, by means of which I was able to show that almost every combination of glass and metal, and also that plain glass disks, when moved and suitably touched, were capable of producing a flow of electricity.

It is one of those combinations, somewhat modified and extended, which $I$ have now the further pleasure of bringing to your notice.

The machine consists of two disks of plate-glass, each of $3 \mathrm{ft} .5$ in. diameter and $\frac{1}{4}$ inch thickness, mounted about $\frac{3}{4}$ inch apart on one boss and spindle. This spindle is driven by means of a haudle, and the disks rotate in one direction.

In the space between the disks are fitted four vertical slips of glass, two being situated on the right-hand side of the machine and two on the left-hand.

The vertical edges of the slips which come nearest the spindle are cut to an angle, leaving a rather wider opening

* Communicated by the Physical Society : rend June 23, 1893. 
at the circumference than at the centre of the disk. Upon each slip is a brush, A, and also an inductor, the brush and

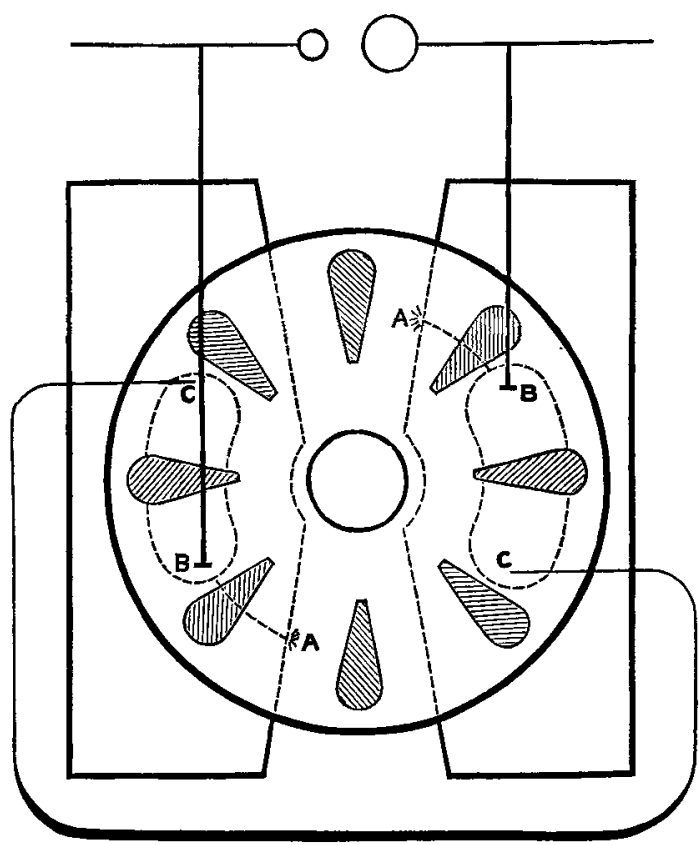

A, A. Brushes connected metallically to the inductors.

B, B. Collecting-brushes connected with terminals.

C, C. Brushes connected with the neutralizing circuit.

the inductor being metallically connected; the brushes are made of fine wire, and touch very lightly on the inner surfaces of the disks. The glass slips slide into place by means of suitable grooves at their top and bottom ends ; they may be removed and replaced readily at pleasure.

The essential parts are fitted together within a glass case, and in all respects the machine is so constructed as to be useful for experimental work. The limited size of the case causes the insulating distances between part and part to be small, hence the length of the sparks is reduced. All the surfaces are coated with shellac, and when the brushes are new and in proper condition the machine is self-exciting.

The charges are not subject to reversal when the terminals are opened beyond the striking distance, for then the whole of the induced charges pass by way of the neutralizing brushes C, C. Another feature is that the neutralizing current may also be broken without reducing the excitement,

Phil. Mag. S. 5. Vol. 36. No. 220. Sept. $1893 . \quad$ T 
but then the charges alternate from positive to negative with each half revolution of the disks.

An approximate measure of the efficiency of the machine is seen by the following experiments.

The glass throughout was held free from electrical excitement, and the disks were rotated sixty times per minute ; the actuating cause was then removed, and the disks came to rest after making forty-seven revolutions. This measures the friction of the machine.

The disks were then similarly turned, but the electrical charges were allowed to collect; it was then found that the disks came to rest after twenty-three revolutions. The friction of the machine, therefore, is about equal to the repulsion of the electrical charges.

Leyden jars having eight square inches of tinfoil in each coating were then connected to the terminals, and the terminals set $3 \frac{1}{2}$ in. apart: the disks were turned to the same number of revolutions and then left to come to rest, which they did after producing eighty sparks.

The disks were then turned and the number of sparks counted in relation to each revolution. This gave seven sparks of $1 \mathrm{in.}$ length, five of $2 \mathrm{in}$., four of $3 \mathrm{in}$., and three of $4 \frac{1}{2}$ in. length.

Eight metal sectors were then added (total 112 square inches on each disk): with them the self-excitement becomes so free that, before any circuit was made, presenting the fingers to the disks caused electrical discharges.

When the circuits are made between the several brushes the disks appear to be seething with electricity, and the charges shoot out for a considerable part of their circumference, but chiefly at the top and the bottom, where they are not covered by the vertical glass slips, for the change of the charges from + to - takes place upon the disks just as they pass the edge of the slip. Improvements in this respect might be made, but the present arrangement has the advantage of giving permanence to the respective charges.

It will be noticed that strips of tinfoil are placed over the receding surfaces of the glass slips. They were intended to afford the means for collecting and carrying to earth any part of the charges from the inductors, but the earth connexion has not been necessary.

When at work the charges passing from the disks to the brushes may be heard some considerable distance; they resemble the beating of the floats of a paddle-wheel.

The charges are, however, reduced about 25 per cent. by the addition of the sectors. 
Further tests have been made by fixing a pulley, 3 in. in diameter, to the overhanging end of the spindle, and suspending therefrom by a cord a weight of 15 lbs. The fall of this weight through three feet produced seventeen revolutions when the disks were not excited and ten revolutions when excited. The same Leyden jars were then connected, and it was found that the fall of the weight through three feet produced twenty-eight sparks of $3 \frac{1}{2}$ in. length. Reducing this to the terms of work in relation to sparking length, and omitting friction of the machine, it will be seen that $1 \mathrm{lb}$. weight falling through rather less than twelve inches produces a spark of $3 \frac{1}{2}$ in. length. It must not, however, be forgotten that a considerable amount of electricity is also passing by way of the neutralizing circuit.

\section{An Influence Machine. By W. R. PIdgeon*.}

TF we follow the action of any single sector on a disk of 1 one of Mr. Wimshurst's beautiful machines, we find it goes through the following electrical changes :-Suppose it to be just leaving the positive collector $\mathrm{C}$, it comes into a

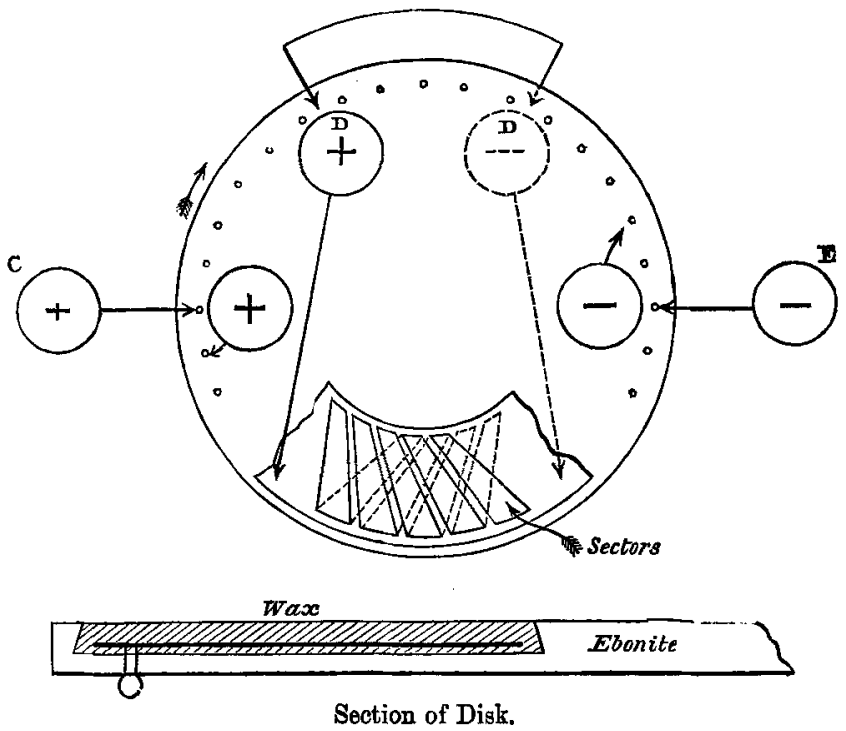

strong positive field produced by the other disk, and while in this field is earthed by the first neutralizing brush $\mathrm{D}$ and

* Communicated by the Physical Society : read June 23, 1893. 